\title{
On the one-phase reduction of the Stefan problem with a variable phase change temperature
}

\author{
T.G. Myers ${ }^{\mathrm{a}, \mathrm{b}}$, F. Font ${ }^{\mathrm{a}, \mathrm{b}}$ \\ ${ }^{a}$ Centre de Recerca Matemàtica, Edifici C, 08193 Bellaterra, Barcelona, Spain \\ ${ }^{b}$ Dept Matemàtica Aplicada I, U. Politècnica de Catalunya, Barcelona, Spain
}

\begin{abstract}
The one-phase reduction of the Stefan problem, where the phase change temperature is a variable, is analysed. It is shown that problems encountered in previous analyses may be traced back to an incorrectly formulated Stefan condition. Energy conserving reductions for Cartesian, cylindrically and spherically symmetric problems are presented and compared with solutions to the two-phase problem.
\end{abstract}

Keywords:

Stefan problem; Melting point depression; Energy conservation.

\section{Introduction}

The Stefan problem where the phase change temperature is fixed is a classical example of a moving boundary problem and has been well-studied for more than 100 years. However, with the advent of a number of new technologies, the situation where a material's phase change temperature differs from the standard value is becoming increasingly important. For example, materials made from supercooled liquids are currently used in medicine, defence and aerospace equipment, electronics and sports $[8,15]$. The phase change temperature of supercooled liquids can vary because the liquid molecules have lower energy than when solidifying under normal circumstances and this affects their ability to move to the solid interface. Nanoparticles have a vast array of applications in medicine, environmental remediation, materials and energy [7]. A key factor in understanding the melting of nanoparticles is the large decrease in melt temperature with decreasing size, for example 
a $2 \mathrm{~nm}$ radius gold nanoparticle will melt at approximately $500 \mathrm{~K}$ below the bulk melt temperature [3]. In this case the high curvature of the melt interface leads to a large value for the surface tension induced stress which then reduces the melt temperature.

In order to simplify the mathematical description of the phase change process it is common to neglect one of the material phases, to produce the one-phase Stefan problem. When the melt temperature is the standard (or homogeneous) phase change temperature, here denoted $T_{m}^{*}$, then the onephase problem is usually well-defined. However, when the phase change temperature is variable then difficulties arise (for example, energy may not be conserved) $[6,13,19]$. The issue with the one-phase formulation has been investigated by looking at asymptotic limits of low thermal conductivity in the solid (compared to that in the liquid) [6] and large conductivity in the solid [13].

In this paper we will demonstrate that problems with the one-phase reduction may arise due to inconsistent assumptions concerning the temperature in the neglected phase. If the reduction is carried out consistently then there is no problem with the energy conservation. The one-phase reduction is invoked to simplify the analysis, another standard simplification involves assuming constant thermal properties throughout the process. If we consider the ratio of the thermal conductivity of water to ice $k=k_{s} / k_{l} \approx 4$ and the specific heat ratio $c=c_{s} / c_{l} \approx 0.5$ then it is clear that this assumption can lead to significant errors. Consequently in the following we will work with different (constant) values in each phase. The density also varies, usually to a lesser extent than conductivity and specific heat [1]. If we include density change in our analysis then the governing equations become more complex, with the addition of advection and kinetic energy, see [9]. Consequently, to keep down the number of terms in the equations and so simplify the arguments we will focus on the situation where the density, $\rho$, is constant throughout the process. However, the arguments may be easily adapted to include it using the equations described in [9].

\section{Governing equations for phase change}

We will now derive the Stefan condition and heat equations for a onedimensional Cartesian problem via an energy balance. For simplicity we examine the case of fixed density and so avoid the velocity terms caused by the shrinkage or expansion of the material. 
The governing equations for the Stefan problem may be obtained from the energy conservation equation

$$
\frac{\partial}{\partial t}\left[\rho I^{*}\right]=-\nabla \cdot \mathbf{q}^{*}
$$

where $\rho$ is the density, $I^{*}$ the internal energy and the conductive heat flux $\mathbf{q}^{*}=-k \nabla T^{*}$. This simply states that internal energy varies with time due to heat movement through the boundary. The star superscript indicates dimensional variables. The internal energy/unit mass is

$$
I_{s}^{*}=c_{s}\left(\theta^{*}-T_{m}^{*}\right) \quad I_{l}^{*}=c_{l}\left(T^{*}-T_{m}^{*}\right)+L_{f},
$$

where subscripts $s, l$ denote solid and liquid, $\theta^{*}, T^{*}$ denote the respective temperatures. The heat equations may be obtained from the energy balance by simply substituting for $I^{*}$ and $\mathbf{q}^{*}$ in (1)

$$
\begin{aligned}
\frac{\partial}{\partial t^{*}}\left[\rho c_{s}\left(\theta^{*}-T_{m}^{*}\right)\right] & =\frac{\partial}{\partial x}\left(k_{s} \frac{\partial \theta^{*}}{\partial x^{*}}\right) \\
\frac{\partial}{\partial t^{*}}\left[\rho\left(c_{l}\left(T^{*}-T_{m}^{*}\right)+L_{f}\right)\right] & =\frac{\partial}{\partial x}\left(k_{l} \frac{\partial T^{*}}{\partial x^{*}}\right) .
\end{aligned}
$$

Noting that all thermal properties and $T_{m}^{*}$ are constant within each phase leads to the familiar form

$$
\rho c_{s} \frac{\partial \theta^{*}}{\partial t^{*}}=k_{s} \frac{\partial^{2} \theta^{*}}{\partial x^{* 2}} \quad \rho c_{l} \frac{\partial T^{*}}{\partial t^{*}}=k_{l} \frac{\partial^{2} T^{*}}{\partial x^{* 2}} .
$$

The Stefan condition may also be obtained from the conservation equation (1) via the Rankine-Hugoniot condition

$$
\frac{\partial f}{\partial t}+\nabla \cdot \mathbf{g}=0 \Rightarrow[f]_{-}^{+} s_{t}=[\mathbf{g} \cdot \mathbf{n}]_{-}^{+},
$$

where $\mathbf{n}$ is the unit normal (in this case it is simply $\hat{\mathbf{x}}$ ) and $f, \mathbf{g}$ are functions evaluated on either side of the discontinuity, $x^{*}=s^{*}\left(t^{*}\right)$. For the case where a fluid initially occupying $x^{*} \geq 0$ is solidified from the boundary $x^{*}=0$ we take the ${ }^{+}$superscript to indicate fluid, $x^{*}>s^{*}$, and ${ }^{-}$to indicate solid, $x^{*}<$ $s^{*}$. Comparing the energy balance (1) to the Rankine-Hugoniot condition shows $f=\rho I^{*}, \mathbf{g}=\mathbf{q}^{*}$, and the Stefan condition follows from the second of equations (6)

$\rho\left[\left(c_{l}\left(T^{*}\left(s^{*}, t^{*}\right)-T_{m}^{*}\right)+L_{f}\right)-c_{s}\left(\theta^{*}\left(s^{*}, t^{*}\right)-T_{m}^{*}\right)\right] s_{t^{*}}^{*}=-\left.k_{l} \frac{\partial T^{*}}{\partial x^{*}}\right|_{x^{*}=s^{*}}+\left.k_{s} \frac{\partial \theta^{*}}{\partial x^{*}}\right|_{x^{*}=s^{*}}$. 
$\frac{\partial}{\partial t^{*}}\left[\rho c_{s}\left(\theta^{*}-T_{m}^{*}\right)\right]=\nabla \cdot\left(k_{s} \nabla \theta^{*}\right) \quad \frac{\partial}{\partial t^{*}}\left[\rho\left(c_{l}\left(T^{*}-T_{m}^{*}\right)+L_{f}\right)\right]=\nabla \cdot\left(k_{l} \nabla T^{*}\right)$

$$
\rho\left[\left(c_{l}\left(T^{*}\left(s^{*}, t^{*}\right)-T_{m}^{*}\right)+L_{f}\right)-c_{s}\left(\theta^{*}\left(s^{*}, t^{*}\right)-T_{m}^{*}\right)\right] s_{t^{*}}^{*}=-\left.k_{l} \frac{\partial T^{*}}{\partial r^{*}}\right|_{r^{*}=R^{*}}+\left.k_{s} \frac{\partial \theta^{*}}{\partial r^{*}}\right|_{r^{*}=R^{*}},
$$

where the phase change front is now located at $r^{*}=R^{*}$ and temperatures depend on $r^{*}, t^{*}$.

\section{Stefan problem with melting point depression}

The standard two-phase, one-dimensional Cartesian Stefan problem with melting point depression is typically specified by heat equations in the solid and liquid phases and the following Stefan condition

$$
\rho\left[\left(c_{l}-c_{s}\right)\left(T_{I}^{*}(t)-T_{m}^{*}\right)+L_{f}\right] s_{t^{*}}^{*}=-\left.k_{l} \frac{\partial T^{*}}{\partial x^{*}}\right|_{x^{*}=s^{*}}+\left.k_{s} \frac{\partial \theta^{*}}{\partial x^{*}}\right|_{x^{*}=s^{*}},
$$

where $T_{I}^{*}(t)$ is the interface temperature, see $[1,4,6,7,20,13,18,19]$. The variation of $T_{I}^{*}(t)$ may be described by a number of relations. For supercooling models an exponential relation between $T_{I}^{*}$ and $s_{t^{*}}^{*}$ holds. This is frequently linearised for small departures from the bulk phase change temperature so $T_{I}^{*}-T_{m}^{*} \propto s_{t^{*}}^{*}[5,8]$. With high curvature some form of GibbsThomson relation is typically used $[7,8]$.

In order to follow previous asymptotic reductions we will now formulate the non-dimensional version of the problem via the following scales,

$$
\theta=\frac{\theta^{*}-T_{m}^{*}}{\Delta T^{*}} \quad T=\frac{T^{*}-T_{m}^{*}}{\Delta T^{*}} \quad x=\frac{x^{*}}{L} \quad t=\frac{t^{*}}{\tau}
$$

where $\Delta T^{*}$ is a temperature scale and $\tau$ the time-scale. In the Stefan problem without melting point depression the length-scale $L$ may be unknown. With melting point depression $L$ may be specified according to the equation governing the phase change temperature. Choosing the time-scale for heat flow in the liquid, $\tau=\rho c_{l} L^{2} / k_{l}$, the heat equations now reduce to

$$
\frac{\partial \theta}{\partial t}=\frac{k}{c} \frac{\partial^{2} \theta}{\partial x^{2}} \quad \frac{\partial T}{\partial t}=\frac{\partial^{2} T}{\partial x^{2}} .
$$


The Stefan condition becomes

$$
\left[(1-c) T_{I}(t)+\beta\right] s_{t}=-\left.\frac{\partial T}{\partial x}\right|_{x=s(t)}+\left.k \frac{\partial \theta}{\partial x}\right|_{x=s(t)},
$$

where $\beta=L_{f} /\left(c_{l} \Delta T\right), k=k_{s} / k_{l}, c=c_{s} / c_{l}$.

These equations are often simplified via a one-phase approximation. Say, for example we neglect the solid phase, then we only need to solve the heat equation in the liquid while the Stefan condition becomes

$$
\left[(1-c) T_{I}(t)+\beta\right] s_{t}=-\left.\frac{\partial T}{\partial x}\right|_{x=s} .
$$

The most familiar form of Stefan condition may be obtained by neglecting melting point depression, so setting $T_{I}=0\left(T_{I}^{*}=T_{m}^{*}\right)$ in equation (14). Equation (14) may also be obtained by choosing $\theta(x, t)$ to be constant or a function of time. Wu et al [19] discuss papers where the solid phase is simply ignored, see [17, 21]. Many authors assume $\theta(x, t)=T_{I}(t)[4,5,10]$ or alternatively $\theta(x, t)=0$ [11]. The first choice has the problem that it does not satisfy the heat equation, whilst the second does not satisfy the interface boundary condition. A more formal way to reduce the system is to let $k=0$, so the liquid conducts heat infinitely faster than the solid. Then the solid temperature is removed from the Stefan condition while the heat equation in the solid becomes $\theta_{t}=0$ and so $\theta$ may be set as a function of $x$ : in practice it is usually taken as 0 or the initial temperature $\theta(x, 0)=\theta_{0}$.

Evans and King [6] discuss a number of papers where the Stefan problem is incorrectly formulated and discuss in detail the approximation where (14) with $T_{I}=0$ is used in conjunction with melting point depression. They point out that this form is popular since it arises in the case without supercooling and is accurate in the limit of large Stefan number. It may also be derived from (13) with the common assumption that $c=1$ and then choosing $k=0$ to remove the contribution of the solid phase. Wu et al [19] discuss similar reductions in the context of spherical nanoparticle melting. They state that when the initial temperature is different to the phase change temperature then the one-phase limit may only be derived under the assumption $k \ll 0$.

In [6] it is stated that the supercooled Stefan problem using (14) with $T_{I}=0$ does not conserve energy. To understand this statement consider the total heat in the system

$$
E=\int_{0}^{s} c \theta d x+\int_{s}^{\infty} T d x
$$


(note, we have chosen $T_{m}^{*}$ as the reference temperature where $E=0$ ). The rate of change of energy is

$$
\frac{\partial E}{\partial t}=\int_{0}^{s} c \frac{\partial \theta}{\partial t} d x+c \theta(s, t) s_{t}+\int_{s}^{\infty} \frac{\partial T}{\partial t} d x-T(s, t) s_{t} .
$$

Replacing the time derivatives via the heat equations and integrating gives

$$
\frac{\partial E}{\partial t}=\left.k \frac{\partial \theta}{\partial x}\right|_{s}-\left.\frac{\partial T}{\partial x}\right|_{s}-\left.k \frac{\partial \theta}{\partial x}\right|_{0}+[c \theta(s, t)-T(s, t)] s_{t},
$$

where we have assumed $T_{x} \rightarrow 0$ as $x \rightarrow \infty$. The standard Stefan condition $\left.T_{x}\right|_{x=s}=-\beta s_{t}$ may be obtained by setting $c=1, \theta(x, t)=T_{I}(t)$ in (13). At the interface $\theta(s, t)=T(s, t)=T_{I}(t)$ and the above energy balance reduces to

$$
\frac{\partial E}{\partial t}=\beta \frac{\partial s}{\partial t}
$$

This equation states that the rate of change of energy balances the heat released by the phase change, so in fact the standard Stefan condition may be consistent with energy conservation (although the choice $\theta(x, t)=T_{I}(t)$ does not satisfy the heat equation). However, if we arrive at the standard Stefan condition by setting $c=1, \theta(x, t)=0$ in equation (13) then the energy balance gives

$$
\frac{\partial E}{\partial t}=\left[\beta-T_{I}\right] s_{t}
$$

and now energy is not conserved (but the heat equation is satisfied). So in fact using the standard Stefan condition it is possible to conserve energy in the system, provided the heat equation is not satisfied, conversely the heat equation may be satisfied but then energy is not conserved.

\section{Asymptotic solutions}

The problem of energy conservation has been tackled in a number of papers by making assumptions on the size of the conductivity ratio and seeking a series expansion in the temperature profiles. Most work has focussed on the limit of small solid to liquid conductivity ratio $k \ll 1[2,6,19]$. However, in [13] it was pointed out that due to the way heat is conducted $k_{s}>k_{l}$ and so the limit $k \gg 1$ was investigated, we shall discuss both cases below. 


\subsection{The limit of small conductivity ratio, $k \ll 1$}

The limit of small $k$ was considered in $[2,6,19]$. In non-dimensional form the analysis of [6] simply incorporates a boundary layer in the solid, of thickness $\mathcal{O}(k)$, which allows the solid temperature to change between $T_{I}(t)$ at $x=s(t)$ to the initial temperature $\theta(x, 0)$ in the far-field which, for simplicity, is set to 0 . Their analysis leads to the modified Stefan condition

$$
\left[T_{I}(t)+\beta\right] s_{t}=-\left.\frac{\partial T}{\partial x}\right|_{x=s(t)} .
$$

This is the correct form of Stefan condition for a far-field temperature $\theta=0$ and it conserves energy. However, this solution is far from ideal. For the problem considered in [6] and by many other authors on the solidification of supercooled liquids the solid does not exist at $t=0$, hence $\theta(x, 0)$ is undefined. Once solidification starts the solid forms at temperature $T_{I}(t) \neq 0$. Since the solid is assumed to be a poor conductor (infinitely poor compared to the liquid) there is no mechanism for the far-field to attain zero temperature. Then, there is the physical issue that solids are better conductors of heat than liquids. Hence this condition, although mathematically correct, is not of use for thermal problems.

\subsection{Limit of large conductivity ratio, $k \gg 1$}

Noting that physically $k_{s}>k_{l}$ it makes more sense to look for a large $k$ reduction. Unlike the $k \rightarrow 0$ limit, when $k \rightarrow \infty$ the solid reacts almost instantaneously to the boundary temperature and so $\theta(x, t) \approx T_{I}(t)$.

Now consider the problem where $k \gg 1$ with boundary conditions on the solid $\theta(s, t)=T_{I}(t), k \theta_{x}(0, t)=-Q$. Note, previously we ignored any heat input at the boundary $x=0$ since the solid was deemed an infinitely poor conductor. In fact previous comparisons between one-phase and two-phase approximations have often been made by imposing $\theta_{x}=0$ at the boundary, see $[4,13]$. Now for generality we allow a non-zero heat flux. If we assume $1 / k \ll 1$ and look for a perturbation solution in terms of the small parameter $1 / k$ then to first order the solid temperature is

$$
\theta(x, t)=T_{I}(t)+\frac{1}{k}\left[\frac{\partial T_{I}}{\partial t} \frac{\left(x^{2}-s^{2}\right)}{2}-Q(x-s)\right] .
$$

Using this to determine $\theta_{x}(s, t)$ in the Stefan condition (13) leads to

$$
-c s \frac{\partial T_{I}}{\partial t}+Q+\left[(1-c) T_{I}+\beta\right] s_{t}=-\left.\frac{\partial T}{\partial x}\right|_{x=s} .
$$


This is the appropriate one-phase Stefan condition, correct to first order, in the case where $k$ is large. The version correct to leading order in $1 / k$ has $\theta(x, t)=T_{I}(t)$ and the first two terms of equation (22) are neglected (thus reducing equation (22) to (14). That is the appropriate one-phase Stefan condition, when $k$ is infinite, is given by equation (14). The most significant difference between using the leading order and first order results is the appearance of the time derivative of $T_{I}$. In the linear undercooling case examined in [13] $T_{I}=-s_{t}$ and so the Stefan condition becomes second order in time, rather than the usual first-order equation.

To verify that the new form conserves energy we may substitute for $\theta$ via equation $(21)$ and $T_{x}(s, t)$ via (22) into the energy equation (17) to find

$$
\frac{\partial E}{\partial t}=Q+\beta s_{t}
$$

This equation states that the rate of change of energy balances that released by the phase change and the energy input at the boundary, i.e. this formulation conserves energy. The heat equation is also satisfied by $\theta(x, t)$. Similarly, the leading order solution $\theta(x, t)=T_{I}(t)$ leads to a consistent, energy conserving solution (it satisfies the heat equation since the limit $k \rightarrow \infty$ results in $\theta_{x x}=0$ ). Obviously the leading order solution will be less accurate than the first order approximation.

\section{Formulation via equation $(7)$}

In $\S 2$ we derived the dimensional Stefan formulation (7). If we compare this with the standard Stefan formulation (10), which is used in studies of melting point depression, we can see that (10) follows from equation (7) if the interface temperature of both phases is $T_{I}^{*}\left(t^{*}\right)$. This implicit assumption, that both phases achieve the same temperature at the interface, is the root of the energy conservation problem.

In non-dimensional form equation (7) may be written

$$
[T(s, t)-c \theta(s, t)+\beta] s_{t}=-\left.\frac{\partial T}{\partial x}\right|_{x=s}+\left.k \frac{\partial \theta}{\partial x}\right|_{x=s} .
$$

This equation should be used as the starting point for any one-phase reduction. For example, to retrieve the poor solid conductor model, $k \ll 1$, with initial temperature $\theta_{0}$, we may impose $\theta(x, t)=\theta_{0}$ and so $\theta(s, t)=\theta_{0}$, 
$\theta_{x}=0$. Substituting these values together with $T(s, t)=T_{I}(t)$ into equation (24) gives

$$
\left[T_{I}(t)-c \theta_{0}+\beta\right] s_{t}=-\left.\frac{\partial T}{\partial x}\right|_{x=s(t)} .
$$

If we choose $\theta_{0}=0$ then equation (20) is retrieved (without the need for an asymptotic analysis).

In the limit of large $k$ the solid is a good conductor and so the interface temperature is immediately transmitted through the material, hence $\theta(x, t)=$ $T_{I}(t)$ and again $\theta_{x}=0$. Equation (24) now reduces to

$$
\left[(1-c) T_{I}(t)+\beta\right] s_{t}=-\left.\frac{\partial T}{\partial x}\right|_{x=s} .
$$

These constitute the final two terms on the left hand side of equation (22), which are the leading order terms in the large $k$ expansion. The first two terms of equation (22) arise as a correction for finite $k$ and come from the fact that for finite $k$ the temperature gradient $\theta_{x}(s, t) \neq 0$.

Note, the popular form specified by equation (13) may be derived from equation (24) by setting $T(s, t)=\theta(s, t)=T_{I}(t)$. One-phase reductions must be consistent with this, either by choosing the temperature of the neglected phase to be $T_{I}(t)$ everywhere or by a boundary layer analysis to match the far-field to the interface temperature, as described in $[2,6,13,19]$. On the other hand, any analysis based on equation (13) where the neglected phase is assigned a constant value not equal to $T_{I}$ will be inconsistent and this manifests itself in the fact the energy balance is not satisfied.

\section{Extension to cylindrical and spherically symmetric geometries}

A common physical situation where the phase change temperature varies involves the melting of nanoparticles or nanowires $[7,14,16,19]$. In this case the melting point depression is a consequence of the surface tension induced pressure. The interest in nano melting for a wide variety of practical applications provides us with the opportunity to investigate a different form of Stefan problem to that of previous sections. In keeping with the analyses of $[7,19]$ we will consider the radially symmetric melting of a sphere or cylinder where a fixed temperature is imposed at the outer boundary $T(1, t)=1$. The 
appropriate nondimensional forms of (12) are

$$
\frac{\partial \theta}{\partial t}=\frac{k}{c} \frac{1}{r^{n}} \frac{\partial}{\partial r}\left(r^{n} \frac{\partial \theta}{\partial r}\right), \quad \frac{\partial T}{\partial t}=\frac{1}{r^{n}} \frac{\partial}{\partial r}\left(r^{n} \frac{\partial T}{\partial r}\right),
$$

where the solid occupies $r \in[0, R(t)]$ and the liquid $r \in[R(t), 1]$. The length-scale has been chosen as the initial radius, $R_{0}$, and the temperature scale $\Delta T=T_{H}-T_{m}^{*}$ where $T_{H}$ is the temperature imposed at the surface. The spherically symmetric model corresponds to $n=2$, cylindrical to $n=1$ and $n=0$ gives us a one-dimensional Cartesian model. The appropriate non-dimensional form for the Stefan condition (24) is

$$
[T(R, t)-c \theta(R, t)+\beta] R_{t}=-\left.\frac{\partial T}{\partial r}\right|_{r=R}+\left.k \frac{\partial \theta}{\partial r}\right|_{r=R} .
$$

Following the arguments of $\S 5$ we may immediately write down the one phase reductions for small and large $k$. For $k \ll 1, \theta(x, t)=\theta(R, t)=\theta_{0}$ and equation (28) reduces to the radial form of (25) (with $s$ replaced by $R$ and $x$ by $r$ ). Choosing $\theta_{0}=0$ this is exactly the one-phase limit used in the study of nanoparticle melting of $[2,19]$ and derived through a boundary layer analysis. For infinite $k, \theta(x, t)=\theta(R, t)=T(R, t)=T_{I}$ and the radial version of $(14)$ is obtained. The correction for large but finite $k$ requires solving the solid heat equation in $(27)$, subject to $\theta(R, t)=T_{I}(t), \theta_{r}(0, t)=0$. This leads to

$$
\theta=T_{I}+\frac{1}{k}\left[\frac{c}{n+1} \frac{\partial T_{I}}{\partial t}\left(\frac{r^{2}-R^{2}}{2}\right)\right]+\mathcal{O}\left(k^{-2}\right) .
$$

Then the Stefan condition, correct to $\mathcal{O}\left(k^{-1}\right)$, is

$$
-\frac{c R}{n+1} \frac{\partial T_{I}}{\partial t}+\left[(1-c) T_{I}+\beta\right] R_{t}=-\left.\frac{\partial T}{\partial r}\right|_{r=R} .
$$

Using the definition of total energy

$$
E=\int_{0}^{R} c \theta r^{n} d r+\int_{R}^{1} \operatorname{Tr}^{n} d r
$$

it is a simple matter to show that the above formulae all conserve energy.

In [13] the accuracy of the Cartesian one-phase formulations was discussed in detail. An example was given for the material salol, which has a low value 

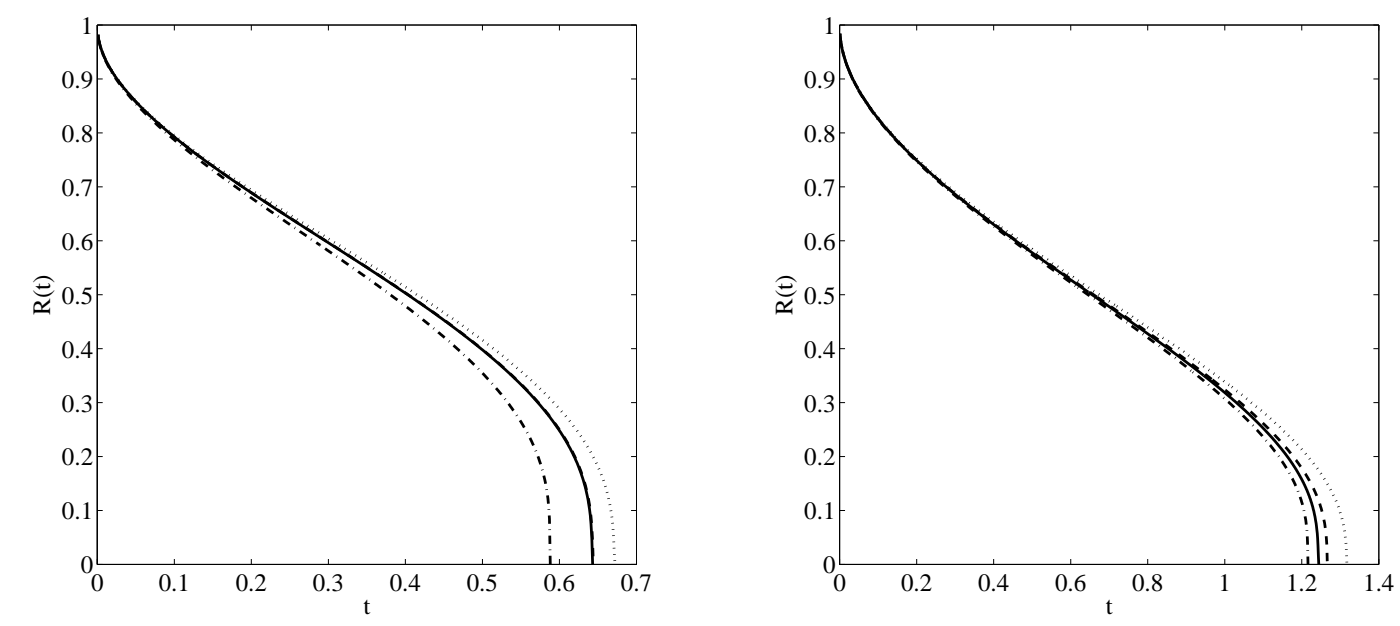

Figure 1: Evolution of the interface $R(t)$ for the two-phase (solid line), large $k$ (dashed), small $k$ (dash-dotted) and standard (dotted) formulations. Plot (a) corresponds to $n=2$ (nanoparticle) and (b) to $n=1$ (nanowire).

of $k \approx 1.3$ and it was shown that the small $k$ solution was far from the twophase solution (with an approximately $40 \%$ slower melt rate). The standard formulation (corresponding to the infinite $k$ limit) was more accurate than the small $k$ solution and the large but finite $k$ result was very accurate. With data appropriate for a water-ice system, $k \approx 4$, the same trend was observed: the small $k$ solution gave the least accurate results, the large $k$ solution was virtually indistinguishable from the two-phase solution. Since the accuracy of the Cartesian model has been established we now focus on the radially symmetric formulations. In Figure 1 we present two sets of results for the evolution of a particle radius for the spherical and cylindrically symmetric models. Parameter values are chosen for gold: $T_{m}^{*}=1337 \mathrm{~K}$, $L_{m} 6.37 \times 10^{4} \mathrm{~J} / \mathrm{kg}, \rho\left(=\rho_{s}\right)=1.93 \times 10^{4} \mathrm{~kg} / \mathrm{m}^{3}, k=2.9906, c=0.7914$, $\sigma_{s l}=0.27 \mathrm{~N} / \mathrm{m}$, see [7]. The melt temperature is described by the standard Gibbs-Thomson relation, $T_{I}(t)=-n \Gamma / R(t)$ where $\Gamma=\sigma_{s l} T_{m}^{*} /\left(R_{0} \rho L_{f} \Delta T\right)$ and $\sigma_{s l}$ is the solid-liquid surface tension. Choosing an initial radius $R_{0}=$ $20 \mathrm{~nm}$ and temperature difference $\Delta T \approx 39 \mathrm{~K}$ we find $\Gamma=0.3755, \beta=10$. Figure 1a shows the melting of a nanosphere, Figure 1b shows a melting nanowire. The four curves in each figure represent the solution of the twophase model of equation (28) (solid line), the large $k$ model of equation (30) 
(dashed line), the standard formulation (or infinite $k$ ) given by the radial version of (14) (dotted line) and the small $k$ model, the radial version of equation (20), (dash-dot line). All curves show the expected feature that for $t \rightarrow 0^{-}$and $R \rightarrow 0^{+}$the velocity $R_{t} \rightarrow-\infty$, see $[2,7,12,18]$. Note, the Gibbs-Thomson relation predicts a negative melt temperature for $R \approx 0.4 \mathrm{~nm}$ while we expect continuum theory to no longer hold below $R=2 \mathrm{~nm}$, see the discussion in $[7,9]$. Consequently the graphs should only be expected to hold for $R>0.1=2 \mathrm{~nm} / 20 \mathrm{~nm}$. In the first graph, for a nanosphere, the large $k$ solution is virtually indistinguishable from the two-phase solution. The dotted line, which represents the infinite $k$ model is close to the twophase solution, while the small $k$ approximation gives the worst result. For the melting of a nanowire, Fig. 1b, again the large $k$ solution is the most accurate but, remarkably, the small $k$ solution shows a similar, if slightly lower, level of accuracy.

\section{Conclusion}

It has been noted by several authors that the standard one-phase reduction to the Stefan problem in the presence of melting point depression does not conserve energy. In this paper we show two important results:

1. Depending on the assumptions made to obtain the standard reduction it may conserve energy (but then the heat equation for the neglected phase is not satisfied).

2. Difficulties encountered in writing down a one-phase reduction which satisfies both energy conservation and the heat equation stem from using the wrong form of Stefan condition, which implicitly incorporates the assumption that both solid and liquid materials are at the phase change temperature at the interface. Provided the correct Stefan condition is used energy conserving forms may be written down immediately. Asymptotic expansions may subsequently be used to improve accuracy.

In the case of melting a semi-infinite solid, in a Cartesian frame, the appropriate two-phase Stefan condition is given by equation (24) and the leading order reduction by equation (26). A reduction accurate to first-order in $1 / k$ is given by equation (22). Similar equations were also derived for spherical and cylindrically symmetric problems.

Obviously there are many varieties of Stefan problem and we have only analysed two particular types of problem. However, their adaptation to many 
other scenarios is straightforward. The key being to start with the correct form of Stefan condition.

\section{Acknowledgments}

The research of TGM was supported by a Marie Curie International Reintegration Grant Industrial applications of moving boundary problems Grant no. FP7-256417 and Ministerio de Ciencia e Innovación Grant MTM201123789. FF acknowledges the support of a Centre de Recerca Matemàtica $\mathrm{PhD}$ grant.

\section{References}

[1] V. Alexiades and A.D. Solomon, Mathematical Modelling of Freezing and Melting Processes, Hemisphere Publishing Corporation, 1st edition, 1993.

[2] J.M. Back, S.W. McCue, M. H-N. Hsieh, T.J. Moroney. Appl. Math. Comp. 229, 41-52, 2014.

[3] P. Buffat, J.P. Borel. Phys. Rev. A 13(6), 228722971976.

[4] C. Charach, B. Zaltzman. Phys. Rev. E., 49(5), 4322-4327, 1994.

[5] S.H. Davis Theory of solidification. Cambridge University Press, 2001.

[6] J. D. Evans, J. R. King. Q.J.M.A.M. 53, 449-473, 2000.

[7] F. Font, T.G. Myers. J Nanopart Res, 15:2086, 2013. DOI 10.1007/s11051-013-2086-3

[8] F. Font, T.G. Myers, S.L. Mitchell. Int. J. Heat Mass Trans. 62, 411-421, 2013.

[9] F. Font, T.G. Myers, S.L. Mitchell. In press Microfluid. Nanofluid.

[10] S.C. Gupta The classical Stefan problem. Elsevier Science B.V. 2003.

[11] L.A. Herraiz, M.A. Herrero, J.J.L. Velzquez. Proc. Roy. Soc. Edin. 131, 371389, 2001. 
[12] R. Kofman, P. Cheyssac, Y. Lereah, A. Stella. Euro. Phys. J. D, 9, 441-444 (1999).

[13] T.G. Myers, S.L. Mitchell, F. Font. Int. Commun. Heat Mass Transf., $39,15221525,2012$

[14] W.H. Qi. Physica B: Condensed Matter, Volume 368(14), pp 46502005

[15] M. Telford. Materials Today, 7(3), 36-43, 2004.

[16] Y-H. Wen, Z-Z. Zhu, R. Zhu, G-F. Shao. Physica E 25(1), pp 4754, 2004

[17] T. Wu, H-C. Liaw, Y-Z. Chen. Int. J. Heat Mass Trans. 45, 2055-2065, 2002.

[18] B. Wu, P. Tillman, S.W. McCue, J.M. Hill. J. Nanosci. Nanotech. 8(1-4) 2008 doi:10.1166/jnn.2008.6017

[19] B. Wu, S.W. McCue, P. Tillman, J.M. Hill. Appl. Math. Model. 33, 23492367, 2009

[20] B. Wu, P. Tillman, S.W. McCue, J.M. Hill. IMA J. Appl. Math. 74, 439457, 2009.

[21] Q. Zhu, A. Peirce, J. Chadam. Euro. J. Appl. Math. 4, 419-436, 1993. 